\title{
Progesterone/RANKL Is a Major Regulatory Axis in the Human Breast
}

\author{
Tamara Tanos, ${ }^{1}$ George Sflomos, ${ }^{1}$ Pablo C. Echeverria, ${ }^{2}$ Ayyakkannu Ayyanan, ${ }^{1}$ \\ Maria Gutierrez, ${ }^{1}$ Jean-Francois Delaloye, ${ }^{3}$ Wassim Raffoul, ${ }^{3}$ Maryse Fiche, ${ }^{3}$ William Dougall, ${ }^{4}$ \\ Pascal Schneider, ${ }^{5}$ Ozden Yalcin-Ozuysal, ${ }^{6}$ Cathrin Brisken $^{1}{ }^{*}$
}

\begin{abstract}
Estrogens and progesterones are major drivers of breast development but also promote carcinogenesis in this organ. Yet, their respective roles and the mechanisms underlying their action in the human breast are unclear. Receptor activator of nuclear factor $\kappa B$ ligand (RANKL) has been identified as a pivotal paracrine mediator of progesterone function in mouse mammary gland development and mammary carcinogenesis. Whether the factor has the same role in humans is of clinical interest because an inhibitor for RANKL, denosumab, is already used for the treatment of bone disease and might benefit breast cancer patients. We show that progesterone receptor (PR) signaling failed to induce RANKL in $\mathrm{PR}^{+}$breast cancer cell lines and in dissociated, cultured breast epithelial cells. In clinical specimens from healthy donors and intact breast tissue microstructures, hormone response was maintained and RANKL expression was under progesterone control, which increased RNA stability. RANKL was sufficient to trigger cell proliferation and was required for progesterone-induced proliferation. The findings were validated in vivo where RANKL protein expression in the breast epithelium correlated with serum progesterone levels and the protein was expressed in a subset of luminal cells that express PR. Thus, important hormonal control mechanisms are conserved across species, making RANKL a potential target in breast cancer treatment and prevention.
\end{abstract}

\section{INTRODUCTION}

Breast cancer is a leading cause of cancer-associated death worldwide. The ovarian hormones estrogens and progesterone control breast development and promote carcinogenesis in this organ (1). Their respective roles are debated, and the mechanisms underlying their action in vivo are poorly understood.

Mouse genetics combined with tissue recombination approaches have revealed that estrogens and progesterone act through their cognate receptors expressed in a subset of mammary epithelial cells to elicit cell proliferation largely by paracrine mechanisms (2). Estrogens drive pubertal development relying on amphiregulin as a paracrine mediator of their proliferative effects $(3,4)$, whereas progesterone is the major proliferative stimulus in the adult mammary gland and acts through both cell-intrinsic and paracrine mechanisms (5).

The tumor necrosis factor (TNF) family member RANKL (receptor activator of nuclear factor $\kappa \mathrm{B}$ ligand), originally identified as a dendritic cell factor and well characterized for its function in osteoclast differentiation (6), is also required for mammary gland development (7). Genetic evidence was provided that RANKL is important as a paracrine mediator of progesterone-induced proliferation in the adult mouse mammary gland $(8,9)$. Systemic inhibition of RANKL signaling by intravenous injection of recombinant osteoprotegerin (rOPG), its decoy receptor, blocked progesterone-induced proliferation in the mammary epithelium (8), suggesting that RANKL may be used as a drug target in the breast epithelium. Furthermore, the factor was suggested

\footnotetext{
'Swiss Institute for Experimental Cancer Research, National Center of Competence in Research Molecular Oncology, School of Life Sciences, Ecole Polytechnique Fédérale de Lausanne, CH-1015 Lausanne, Switzerland. ²Département de Biologie Cellulaire, Université de Genève, Sciences 3, 1211 Genève 4, Switzerland. ${ }^{3}$ Centre Hospitalier Universitaire Vaudois, 1011 Lausanne, Switzerland. ${ }^{4}$ Department of Hematology/Oncology Research, Amgen Inc., Seattle, WA 98119, USA. 'Department of Biochemistry, University of Lausanne, 1066 Epalinges, Switzerland. 'Department of Molecular Biology and Genetics, Izmir Institute of Technology, 35430 Urla, Izmir, Turkey.

${ }^{*}$ Corresponding author. E-mail: cathrin.brisken@epfl.ch
}

to be involved in expansion of mammary stem cells that have been characterized by high cell surface expression of integrins $\alpha_{6}$ (CD49f) and $\beta_{1}(\mathrm{CD} 29)$ by fluorescence-activated cell sorting (FACS) $(10,11)$. The cell populations expressing high levels of CD49f or CD29 are enriched for cells with the ability to reconstitute mammary fat pads divested of the endogenous epithelium in low numbers $(12,13)$. However, the physiologic relevance of multipotent stem cells identified by this experimental approach was questioned by recent publications, suggesting that lineage-restricted stem cells drive normal development $(14,15)$. Beyond these physiological roles, RANK signaling was shown to be important to mammary tumorigenesis; both genetic and pharmacological inhibition interfered with tumor progression in different mammary tumor models $(16,17)$ and metastasis, the latter by a $\mathrm{T}$ cell-dependent mechanism (18). A RANKL-inhibiting antibody, denosumab (Amgen), has been U.S. Food and Drug Administration (FDA)-approved for treatment of postmenopausal women at high risk for fracture and for prevention of skeletal-related events in patients with bone metastases. Hence, whether the findings in the mouse model apply to humans and whether denosumab may have a place in breast cancer prevention and/or treatment is an urgent question.

The hormone receptor-positive breast cancer cell lines MCF-7 or T47D are widely used models to study hormone action and have yielded detailed knowledge on molecular mechanisms underlying estrogen receptor (ER) and progesterone receptor (PR) signaling, respectively (19). However, genes modulated by hormone signaling in these cell lines differ from target genes identified in mice in vivo (20). Primary human breast epithelial cells (HBECs) grown in vitro lose steroid hormone receptor expression, and therefore, they cannot be used to study estrogen and progesterone action. The importance of extracellular matrix, three-dimensional (3D) structure, and matrix stiffness for mammary epithelial cell biology has been well documented $(21,22)$, and the effects of these factors on cell signaling have been extensively characterized. However, even in a sophisticated 3D culture approach in 
which HBECs retain hormone receptor expression, the in vivo downstream targets identified in the mouse mammary gland, Wnt- 4 and RANKL, are not induced by progesterone (23).

This leads to the question of whether different in vitro systems do not adequately reflect the in vivo complexities or whether the discrepancies are due to differences in regulatory networks between humans and mice. Indeed, differences in tissue composition, the physiology of human menstrual and murine estrous cycle, as well as hormone levels may limit the relevance of findings in mice to humans.

Here, we argue that steroid hormone signaling, which relies on paracrine factors, can only be studied adequately in the context of tissue samples with intact architecture. By extension, previous models in which cells are dissociated before being embedded into matrixes failed to preserve this signaling context. On the basis of our hypothesis, we developed an ex vivo model using breast tissue microstructures isolated from fresh clinical specimens that preserve extensive intercellular contacts and contain multiple cell types. We show that these structures remain hormone-responsive; more specifically, progesterone induces cell proliferation with RANKL, whose mRNA stability is increased, as an essential mediator. Our findings suggest that important growth control pathways in the breast are conserved between mouse and human and that PR/RANKL signaling may be targeted in breast cancer prevention and treatment.

\section{RESULTS}

\section{Development of an ex vivo model to study hormone action in the human breast}

To study the role of estrogens and progesterone in the human breast, we collected fresh human breast tissue specimens from 151 patients undergoing reduction mammoplasties after obtaining their informed consent. We obtained their medical and reproductive history and a blood sample to determine serum progesterone levels at the time of surgery (table S1). A biobank was built with tissue stored in paraffin, flash-frozen, and processed for storage in freezing medium. The frozen samples were used to establish the ex vivo approach described hereafter. All experiments shown below were performed with freshly isolated tissue to avoid possible artifacts due to freezing.

We reasoned that it is important to preserve intercellular interactions to study hormone action in the breast epithelium because steroid hormone action relies largely on paracrine signaling (24). Tissue from fresh reduction mammoplasties was mechanically and enzymatically dissociated (Fig. 1A). The resulting tissue fragments of up to $2 \mathrm{~mm}$ in size have ducts and lobules that reflect their anatomic site of origin (Fig. 1B). Hence, we refer to them as "tissue microstructures" in contrast to the term "organoids" that designates tissue fragments isolated from the mouse mammary gland previously used to study progesterone response (25), which are round and amorphous (Fig. 1C). The tissue microstructures were suspended in minimal medium; cell viability remained more than $90 \%$ until after 6 days of culture as assessed by trypan blue staining (Fig. 1D). The breast tissue microstructures derived from seven different patients contained between 1 and 24\% of proliferating cells after 24 and 48 hours, as assessed by immunostaining for the S/M phase-specific marker phospho-histone H3 (Fig. 1E). To exclude the formal possibility that cells were stalled in S/M phase in the ex vivo condition, we exposed freshly isolated tissue microstructures to two different nucleotide analogs; during the first
24 hours of culture, they were exposed to 5-bromo-2'-deoxyuridine (BrdU) and, after washing, to 5-ethynyl-2'-deoxyuridine (EdU) for the subsequent 24 hours. Double immunofluorescence revealed that both analogs were incorporated into distinct cells (Fig. 1F), indicating that cells were synthesizing DNA, hence actively progressing through the cell cycle in the ex vivo model. Thus, in the context of these tissue microstructures, cell proliferation occurred even in the absence of exogenous growth factors. This contrasts with HBECs plated on plastic, which, like cultured cell lines, do not proliferate when kept in growth factor- and serum-free medium (26).

To further characterize the microstructures, we performed immunostaining for the intermediate filaments CK18, specific for luminal cells, and CK14, expressed in basal cells of ducts and both luminal and basal cells in the lobules, as well as the basal/myoepithelial marker p63. The stainings revealed that the major cell types and the bilayered structure of the human breast epithelium are maintained in the ex vivo model (Fig. 1G). Next, we assessed the expression of hormone receptors, which is lost upon in vitro culture of primary HBECs. In the tissue microstructures, about $30 \%$ of the epithelial cells expressed $\mathrm{ER} \alpha$ and PR on day 4 (Fig. 1J), similar to what is observed in the normal human breast (27). To address whether the continued expression of hormone receptors related to the preserved intercellular contacts in the tissue microstructures or to the suspension culture, we dissociated tissue microstructures to single cells and plated them in suspension on low attachment plates. Quantification of PR expression in such samples from three different patients revealed that initially $3.3 \%$ of the cells expressed PR, consistent with a large number of stromal cells in the tissue microstructures. This percentage decreased to $0.4 \%$ on day 4 (Fig. 1, H and I). Thus, suspension culture per se did not prevent loss of hormone receptor expression, but preserving tissue structure is likely important to maintain hormone receptor expression.

To test whether hormone receptor signaling is intact in the ex vivo system, we exposed tissue microstructures for 24 hours to either vehicle or $\mathrm{E}_{2}$ and determined protein levels of the ER $\alpha$ target gene PR. In three patient samples, $P R$ protein levels were increased by the $E_{2}$ treatment (Fig. $1 \mathrm{~K}$ ). In an additional sample, the response to $\mathrm{E}_{2}$ was tested on three subsequent days, and PR protein expression was consistently induced (Fig. 1L). Thus, ER $\alpha$ signaling remains functional for at least 3 days in the ex vivo model.

\section{Cell proliferation in response to activation of ERo and PR signaling}

Next, we examined the effects of ER $\alpha$ and PR signaling activation on cell proliferation. To activate the two pathways, we used $\mathrm{E}_{2}(20 \mathrm{nM})$ and the stable PR agonist R5020 (20 nM), respectively. Tissue microstructures from 29 different patients were processed and stained for phospho-histone H3 after 24 hours of stimulation. Stimulation with $\mathrm{E}_{2}$ did not elicit statistically significant changes in the proliferative index, but tissue microstructures stimulated with R5020 had an average increase of twofold ( $P=0.01$ ) (Fig. 2A). Surprisingly, combined $\mathrm{E}_{2}$ and R5020 treatment did not induce significant increase in cell proliferation $(P=0.16)$.

Human breast tissue samples are very heterogeneous. Within a given breast, cellularity and cell type composition can vary between different sectors. Moreover, histological features differ largely among different patients (fig. S1). Patients were of different ages (17 to 78 years) (table S1) and had distinct reproductive histories and different endocrine milieus at the time of surgery. Moreover, several patients used oral 
A

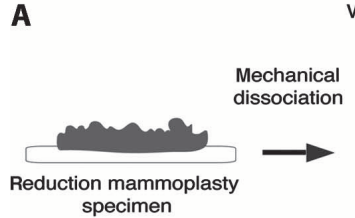
specimen
Vehicle treatment

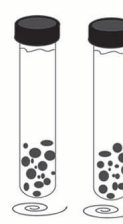

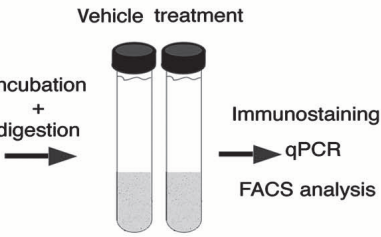

D

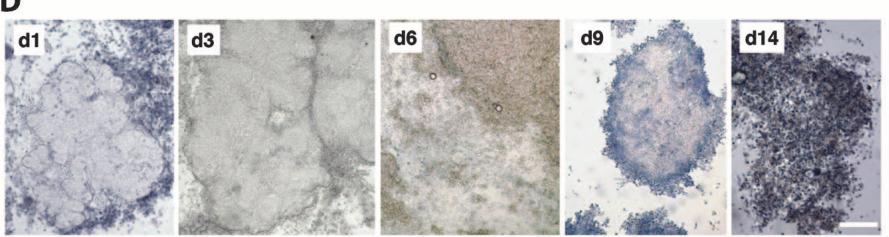

G

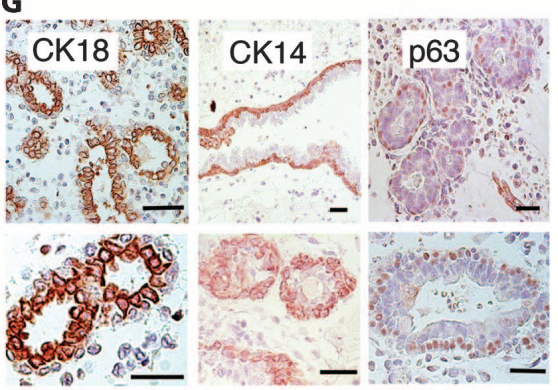

J
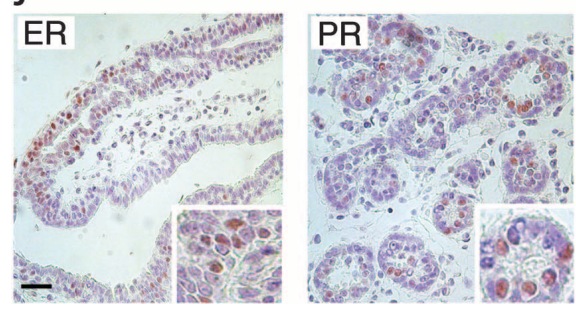

$\mathbf{K}$
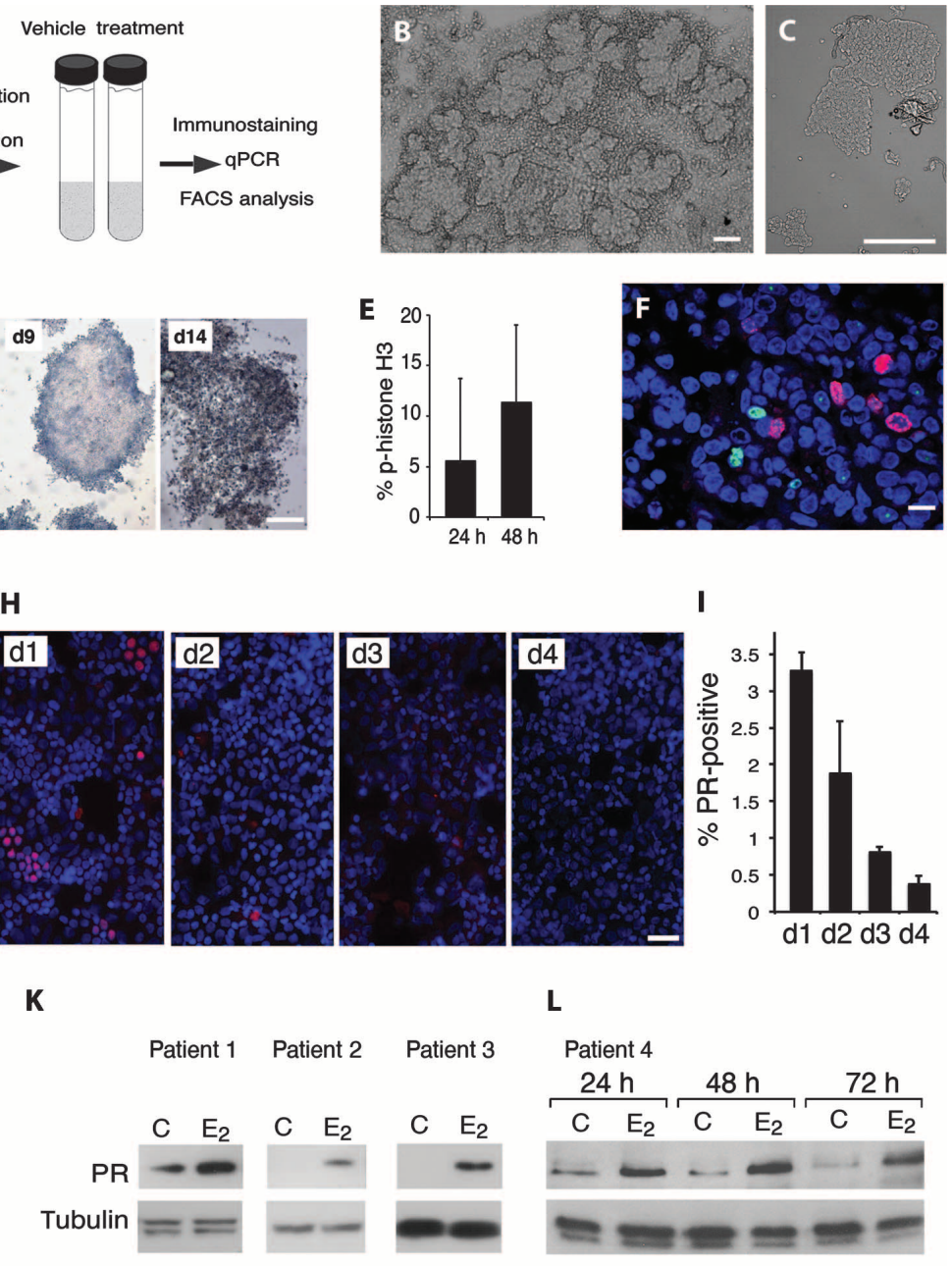

|
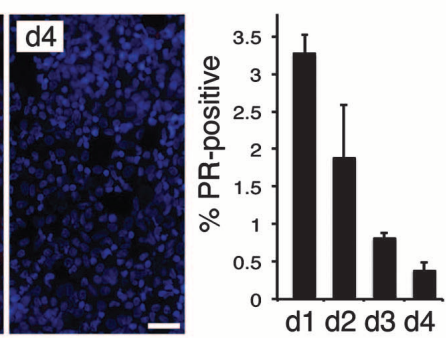

$\mathbf{L}$

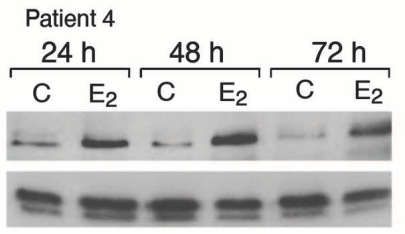

Fig. 1. Ex vivo system for hormone action in the human breast. (A) Scheme of the experimental setup. (B) Human tissue microstructures freshly isolated from reduction mammoplasty specimen. Scale bar, $100 \mu \mathrm{m}$. (C) Mouse organoids freshly isolated from mammary glands. Scale bar, $100 \mu \mathrm{m}$. (D) Ex vivo tissue microstructures in suspension at different days, stained with trypan blue to reveal dead cells. Until day 6, more than $90 \%$ of the cells are viable. Scale bar, $1 \mathrm{~mm}$. (E) Bar plot showing cell proliferation index \pm SD in tissue microstructures cultured without growth factors as determined by phospho-histone $\mathrm{H} 3$ immunostaining 24 and 48 hours after isolation ( $n=7$ for either time point). (F) Anti-BrdU (green) and anti-EdU staining (red) of tissue microstructures that were exposed to BrdU during the first 24 hours in culture and to EdU during the ensuing 24 hours, counterstained with 4',6-diamidino-2-phenylindole (DAPI). Several cells have incorporated either nucleotide. (G) Histological sections of tissue microstructures paraffin-embedded after 48 hours in culture immunostained with anti-cytokeratin 18 (CK18), anti-CK14, and anti-p63 antibodies. Note that the bilayered structure of the human breast epithelium is maintained. Scale bars, $100 \mu \mathrm{m}$. (H) Immunofluorescence for PR (red) counterstained with DAPI (blue) after cytospin of dissociated organoids that were grown in suspension for 24, 48, 72, and 96 hours. Scale bar, $100 \mu \mathrm{m}$. (I) Bar plot showing quantification of $\mathrm{PR}^{+}$cells (mean percentage $\left.\pm \mathrm{SD}\right)(n=3)$. (J) Immunostaining of tissue microstructures on day 4 of culture for ERo and PR. (K) Immunoblots stained with PR and tubulin antibodies on lysates from tissue microstructures from three different patients exposed for 24 hours to either vehicle or $17 \beta$-estradiol $\left(E_{2}\right)$. (L) A fourth patient's sample was exposed to either vehicle or $E_{2}$ on day 1,2 , or 3 of culture. contraception, thereby exposing their breast tissue to different exogenous hormones and their analogs.

We asked whether discrete response patterns might underlie the large variation. Using Cytoscape software $(28,29)$, we organized the samples in a correlation network according to their proliferative responses to $\mathrm{E}_{2}$ and $\mathrm{R} 5020$, alone or in combination, compared to vehicle, as well as the comparison of the response to R5020 to either $\mathrm{E}_{2}$ alone or the combination of the two. Five different groups were distinguished (Fig. 2B); the largest number of samples was characterized by a clear proliferative response to progesterone and an inhibitory effect on cell proliferation by $\mathrm{E}_{2}$ (group 1) (Fig. 2B). Groups 2 and 3 showed increased cell proliferation in response to progesterone but also to $E_{2}$ to different extent. Group 4 comprised nonresponders, and group 5 was characterized by a proliferative response specifically to $\mathrm{E}_{2}$; two of three samples in group 5 were from patients under 20 years of age. No statistically significant correlation between contraceptive pill intake, menstrual cycle status, and parity and the proliferative response to $E_{2}$ and R5020 was found in the present small patient cohort $(n=29)$. 
A

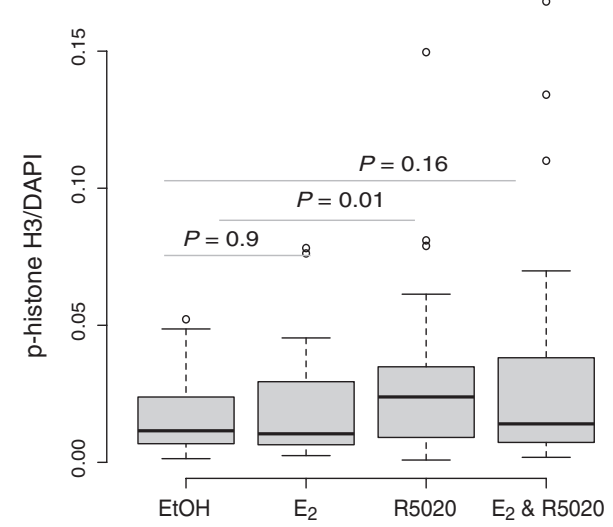

B

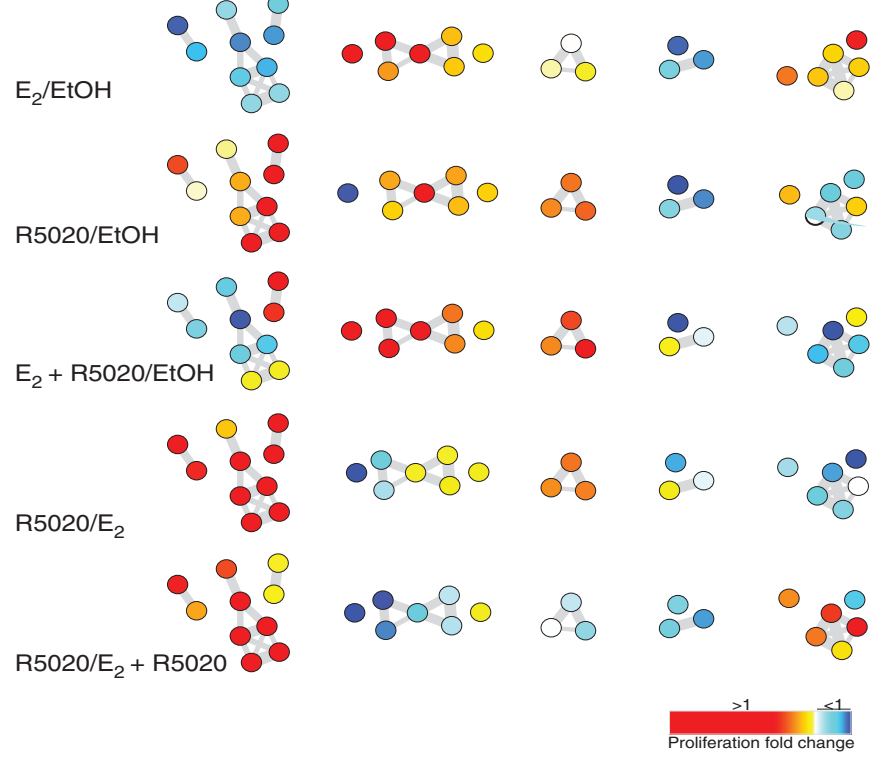

Fig. 2. Hormone-induced cell proliferation in breast tissue microstructures. (A) Box plot showing cell proliferation index as determined by immunofluorescence for phospho-histone $\mathrm{H} 3$ of DAPI-counterstained nuclei in tissue microstructures exposed for 24 hours to vehicle [ethanol (EtOH)], $E_{2}, R 5020$, or $E_{2}$ and R5020. Only R5020 induced statistically significant cell proliferation ( $P=0.01$, two-tailed Student's $t$ test; $n=29$ ). (B) Network correlation organization and analysis of cell proliferation in response to hormone stimulation in 29 patient samples reveals five distinct response patterns (groups 1 to 5) described in the main text. Nodes (samples) were colored with a red-to-blue gradient according to fold change in cell proliferation of pairwise comparisons (see inset for color gradient).

The strongest proliferative response to progesterone (group1) occurred in samples from 35- to 45-year-old patients.

\section{Effects of PR signaling on RANKL expression}

The data indicate that progesterone acts as a proliferative stimulus in the adult human breast. In the mouse mammary gland, progesterone elicits cell proliferation largely by a paracrine mechanism that requires expression of the PR target gene RANKL (8). Treatment of $\mathrm{PR}^{+}$breast cancer cell lines, T47D and MCF-7, as well as primary HBECs with
R5020 did not induce RANKL protein expression (Fig. 3A). Similarly, RANKL mRNA induction had not been detected in primary HBECs grown in Matrigel (23). When R5020 was added to freshly prepared tissue microstructures, RANKL mRNA levels increased $230 \%$ within 4 hours ( $n=11, P=0.003)$ (Fig. 3B). Similarly, RANKL protein levels in tissue microstructures were consistently increased upon 24 hours of R5020 treatment (Fig. 3C). Some induction by $\mathrm{E}_{2}$ was observed in two of three patients tested.

Progesterone and RANKL have been implicated in the control of pluripotent mouse mammary stem cells via RANK, the cognate receptor of RANKL, and RANK mRNA expression was shown to be enriched in the basal cell population of the mouse mammary gland that comprises the stem cells $(10,11)$. In the human breast, stem cells have similarly been shown to reside in the basal compartment, which is characterized by cell surface expression of CD10 (30). To test whether progesterone stimulation results in expansion of the $\mathrm{CD} 10^{+}$cell population, we purified different cell types from tissue microstructures that had been exposed to R5020 or vehicle for 24 hours. The microstructures were dissociated and depleted from immune cells, fibroblasts, and endothelial cells with a cocktail of anti-CD45, anti-Fap, and anti-CD31 antibodies. Subsequently, cells were FACS-sorted on the basis of expression of the pan-epithelial marker EpCAM and CD10. This approach distinguishes three cell populations: $\mathrm{EpCAM}^{\text {high }} / \mathrm{CD} 10^{-}$ enriched for luminal cells, EpCAM ${ }^{\text {low }} / \mathrm{CD} 10^{+}$containing basal cells, and an $\mathrm{EpCAM}^{-} / \mathrm{CD} 10^{-}$double-negative population (Fig. 3D) (30). By this approach, we did not detect significant changes in the ratio of different cell populations upon R5020 stimulation in nine patient samples (Fig. 3E). Quantitative reverse transcription polymerase chain reactions (qRT-PCRs) for myoepithelial-specific p63 and the luminal CK18 mRNA showed expected enrichment in the separated populations, and there were no significant changes in the expression levels of the basal markers CD10 and p63 within the sorted cell populations (Fig. 3F). The mRNA for the cognate RANKL receptor RANK was enriched in the EpCAM ${ }^{\text {high }}$ luminal cell population characterized by CK18 expression (Fig. 3F).

Consistent with RANKL being expressed in $\mathrm{PR}^{+}$luminal cells, its mRNA was enriched in EpCAM ${ }^{\text {high }}$ cells (fig. S2). R5020 stimulation of tissue microstructures increased RANKL mRNA levels up to 40-fold in the EpCAM high populations of the three patient samples analyzed (Fig. 3G). Similarly, another progesterone target gene well established in the mouse mammary gland, Wnt-4 (31), which, like RANKL, was not induced in the in vitro Matrigel system (23), was induced up to sevenfold in the EpCAM ${ }^{\text {high }}$ population after 24 hours of progesterone stimulation (Fig. 3G). Thus, two essential in vivo PR targets, RANKL and Wnt-4, are induced in the tissue microstructures by activation of PR signaling in human luminal breast epithelial cells.

\section{Role of RANKL in progesterone-induced cell proliferation}

To test whether RANKL stimulation is sufficient to induce cell proliferation, we added recombinant RANKL (rRANKL) protein $(1 \mu \mathrm{g} / \mathrm{ml})$ to tissue microstructures from 16 different patients for 24 hours. Immunostaining for RANKL on fixed tissue microstructures detected RANKL protein without tyramide signal amplification (TSA) required to detect endogenous RANKL expression (Fig. 4, A and B), indicating that the rRANKL reaches cells inside the tissue microstructures. RANKL stimulation induced a 2.5 -fold increase in the proliferative index as assessed by phospho-histone $\mathrm{H} 3$ staining $(n=16, P<0.02)$ (Fig. $4 \mathrm{C}$ ), and this occurred in a dose-responsive manner (Fig. 4D). 
A

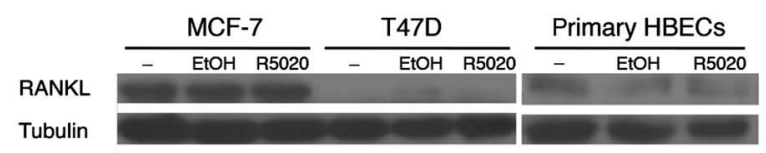

C

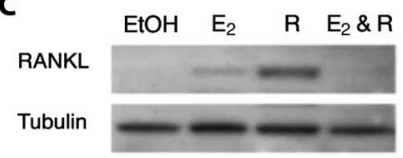

D

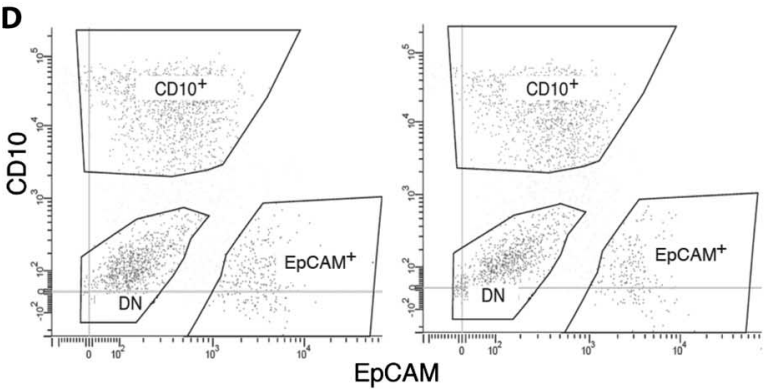

$\mathbf{F}$
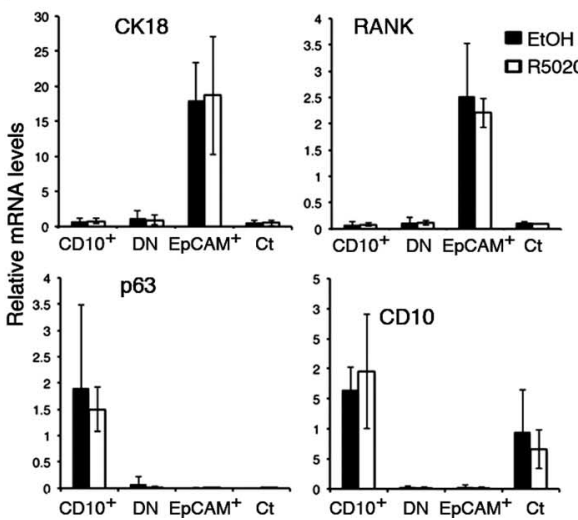

Fig. 3. Control of RANKL expression by PR signaling. (A) Immunoblot of MCF-7, T47D, and primary HBECs cultured on plastic dishes untreated or exposed to either vehicle (EtOH) or R5020 for 24 hours. RANKL protein levels are not affected by R5020. (B) Relative RANKL mRNA levels normalized to hypoxanthine-guanine phosphoribosyltransferase (HPRT) mRNA were assessed by RT-PCR in tissue microstructures from 10 different patients that were treated with vehicle or R5020 for 4 hours. Large black dots represent means \pm SD. (C) Immunoblot of protein lysates from tissue microstructures exposed for 24 hours to either vehicle (EtOH), $E_{2}$, R5020 (R), or $E_{2}$ and R5020 ( $\left.E_{2} \& R\right)$, representative of three independent experiments. (D) FACS scattergrams showing different epithelial cell populations isolated from tissue microstructures after 24 hours of incubation with vehicle or R5020. Stromal cells were first depleted with anti-CD31, anti-CD45, and anti-Fap antibodies (cocktail), and remaining cells were stained with anti-CD10 and anti-EpCAM antibodies. (E) The ratio between the different cell populations $\pm \mathrm{SD}$ in tissue microstructures is not significantly affected by the R5020 treatment $\left[n=9\right.$; $\mathrm{EpCAM}^{+}, P=0.77 ; \mathrm{CD}^{+} 0^{+}, P=0.81$; double-negative (DN), $\left.P=0.81\right]$. (F) Marker gene expression in FACS-sorted cell populations from dissociated breast tissue microstructures exposed for 24 hours to either vehicle (closed bars) or R5020 (open bars). Relative mRNA expression levels of CK18, RANK, p63, and CD10 were normalized to HPRT. Graphs represent means \pm SD of independent experiments performed on tissue from four different patients. Note that RANK mRNA expression is enriched in the EpCAM ${ }^{+}$ luminal cell compartment. (G) Bar plots showing relative mRNA expression levels of RANKL (left) and Wnt-4 (right) normalized to HPRT in FACS-sorted $\mathrm{EpCAM}^{+}$cells from tissue microstructures exposed for 24 hours to either vehicle $(\mathrm{EtOH})$ or R5020. Experiment is representative of three independent experiments. absence of rOPG. Analysis of three different patients showed that addition of $\mathrm{rOPG}$ $(10 \mu \mathrm{g} / \mathrm{ml})$ abrogated, on average, $80 \%$ of the R5020-induced cell proliferation $(P=$ 0.01 ) (Fig. 4E). The basal proliferative indices differ substantially between patients (Fig. 4E). In three additional patient samples, a higher dose of rOPG $(20 \mu \mathrm{g} / \mathrm{ml})$ was included, which showed a stronger inhibition (Fig. 4F). Similar results were obtained at 48 hours $(n=3)$ (Fig. 4G). Thus, RANKL is sufficient to induce proliferation of HBECs and is required for PR signalinginduced cell proliferation.

\section{RANKL expression in vivo}

To assess whether the observations in the ex vivo model are relevant to the human breast in vivo, we used the biobank of reduction mammoplasty specimens $(n=$ 151) (table S1). More specifically, we identified 16 patients who had high serum progesterone levels when their breast tissue was surgically removed. We were able to age-match 10 of these patients with patients who had low progesterone levels and did not take exogenous hormonal preparations. In the paraffin-embedded breast tissue samples from these 10 control samples, that is, women with low serum progesterone levels $(<4 \mathrm{nM})$, we failed to detect RANKL protein by immunohistochemistry. The protein was readily detected in all 10 samples derived from women with high serum progesterone levels (15 to $62 \mathrm{nM}$ ) (Fig. 5, A and B). Furthermore, during pregnancy, when progesterone levels increase further $(>300 \mathrm{nM})$, RANKL expression was particularly high, with more widespread expression and a stronger fluorescence signal (Fig. 5, A and B). Thus, RANKL protein expression correlates with serum progesterone levels in the human breast in vivo.

Double immunofluorescence for RANKL and PR revealed that between 51 and $78 \%$ of the $\mathrm{PR}^{+}$cells showed detectable RANKL expression (Fig. 5, C and D). Conversely, on average, $97 \%$ of $\mathrm{RANKL}^{+}$ cells show detectable PR expression (Fig. 5, C and D). Together, our data strongly support the hypothesis that RANKL expression is controlled by progesterone in the human breast epithelium in vivo.
To address whether the proliferative effects of progesterone require RANKL signaling, we made use of the decoy RANKL receptor OPG. Tissue microstructures were stimulated with R5020 for 24 hours in the presence or

\section{Control of RANKL mRNA expression by progesterone}

Having ascertained that control of RANKL expression by progesterone in the breast epithelium is conserved between mice and humans, 

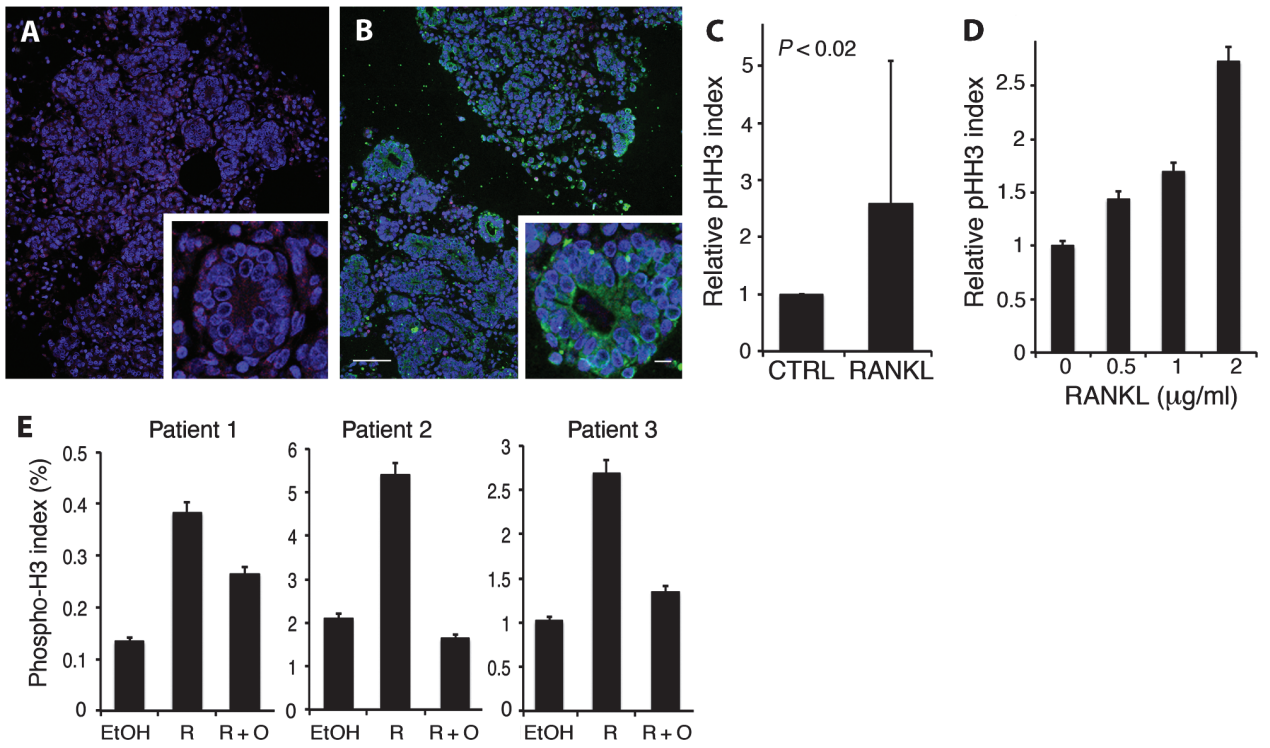

$\mathbf{F}$
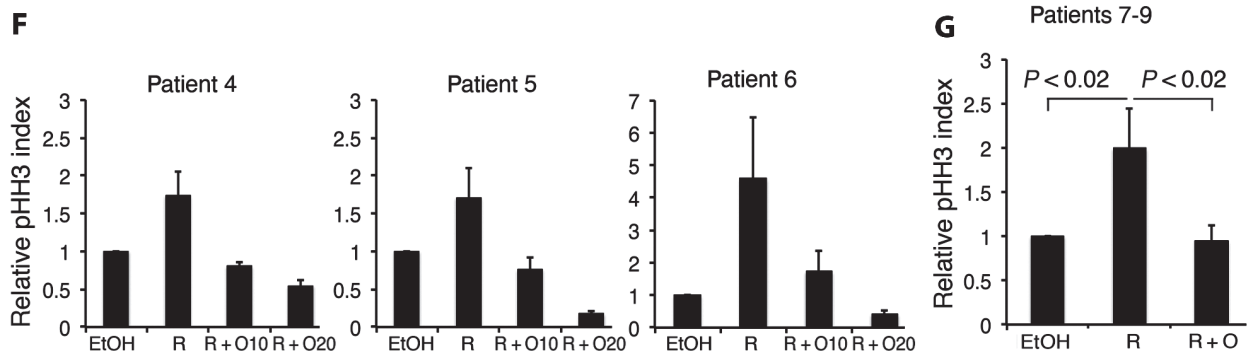

Fig. 4. RANKL and $P R$ signaling and cell proliferation. ( $A$ and $B$ ) Anti-RANKL immunostaining of human tissue microstructures exposed to vehicle (A) or rRANKL $(1 \mu \mathrm{g} / \mathrm{ml})$ (B) without TSA amplification, representative of three independent samples. Scale bars, $100 \mu \mathrm{m}$ (inset, $20 \mu \mathrm{m}$ ). (C) Bar graph showing relative phospho-histone $\mathrm{H} 3(\mathrm{pHH} 3)$ index $\pm \mathrm{SD}$ in tissue microstructures from 16 different patients treated with vehicle (CTRL) or rRANKL $(1 \mu \mathrm{g} / \mathrm{ml})$. (D) Bar graph showing relative phospho-histone $\mathrm{H} 3$ index after 24 hours of stimulation with different doses of rRANKL, representative of three independent experiments. (E) Bar plots showing phospho-histone $\mathrm{H} 3$ index in tissue microstructures isolated from three different patients after 24 hours of treatment with vehicle (EtOH), R5020 (R), or R5020 and rOPG $(10 \mu \mathrm{g} / \mathrm{ml})(\mathrm{R}+\mathrm{O})$. (F) Bar plots showing relative phospho-histone $\mathrm{H} 3$ index in tissue microstructures isolated from three different patients after 24 hours of treatment with vehicle, R5020, or R5020 and rOPG (10 $\mu \mathrm{g} / \mathrm{ml})(010)$ or rOPG $(20 \mu \mathrm{g} / \mathrm{ml})(\mathrm{O} 20)$. SD reflects counts taken on different sections of the same sample. (G) Bar plots showing relative phospho-histone $\mathrm{H} 3$ index $\pm \mathrm{SD}$ in tissue microstructures isolated from three different patients after 48 hours of treatment with vehicle (EtOH), R5020, or R5020 and rOPG $(10 \mu \mathrm{g} / \mathrm{ml})$. independent of R5020 (Fig. 6C). Next, we quantified the levels of the primary transcript by real-time RT-PCR with primers spanning the first exon-intron junction and the mature transcript, using primers within different exons (Fig. 6E, P0 and M0). Organoids that had been stimulated with R5020 for 4 hours showed, on average, a 37 -fold increase in the levels of the mature message, whereas the levels of the primary transcript increased only twofold in three independent experiments (Fig. 6D). We designed more primer sets in which an exon-specific primer is shared and the second primer is either intron-specific (primary transcript) or exon-specific (mature transcript) and chose three primer sets that amplified the two transcripts with equal efficiency (Fig. 6E). Again, the primary transcript was, on average, induced 2.2fold, whereas the mature transcript was induced 9.1-fold. Thus, in mouse mammary organoids, PR signaling controls RANKL mRNA levels predominantly at the mRNA maturation/stability rather than at the transcriptional level.

To assess whether the same applies to human breast epithelium, we used FACS sorting and enriched with EpCAM for PR/RANKL-expressing cells. We selected three distinct primer pairs for primary and mature transcript of equal amplification efficiency and made use of microfluidics (Fluidigm) to analyze the expression in the EpCAM ${ }^{\text {high }}$ cells from stimulated tissue microstructures from three different patients. Expression of the primary transcript was induced, on average, 1.7-fold, whereas the mature transcript was induced 7.5-fold (Fig. 6E), suggesting that also in the human breast, RANKL mRNA expression is controlled, at least partially, by posttranscriptional mechanisms. we went back to the inbred mouse model to determine the mechanisms by which progesterone signaling affects RANKL expression. Treatment of freshly isolated mammary organoids with R5020 led to an average $400 \%$ increase in RANKL mRNA levels within $30 \mathrm{~min}$ (Fig. 6A). RANKL may be a direct transcriptional target of PR signaling; however, there are no well-defined PR binding sites in the RANKL promoter. To address whether R5020 affects the half-life $\left(T_{1 / 2}\right)$ of RANKL mRNA, we treated primary mouse mammary epithelial cells with the transcription inhibitor actinomycin in the presence or absence of R5020. In the absence of R5020, the $T_{1 / 2}$ of RANKL mRNA was 1 hour, whereas in the presence of R5020, the levels of RANKL mRNA were more than $90 \%$ of the pretreatment levels after 2 hours (Fig. 6B), indicating a $T_{1 / 2}$ of more than 6 hours. Control mRNAs such as CK14, CK18, and 36B4 had half-lives greater than 6 hours

\section{DISCUSSION}

The goal of this study was to address the long-standing need for a model system to study hormone action in the human breast and to determine what controls cell proliferation in the human breast epithelium. We developed an ex vivo model based on tissue microstructures isolated from fresh breast specimens that maintains the cellular organization of breast epithelium, expression of ER $\alpha$ and PR, and responsiveness to hormones. Using this model, we demonstrate that PR signaling induces cell proliferation. This is consistent with the clinical observation that cell proliferation occurs during the luteal phase when serum progesterone levels are elevated (32). In this system, two important mediators of progesterone action identified in the mouse, RANKL and Wnt-4 $(8,31,33)$, are induced by PR signaling. RANKL is required and 

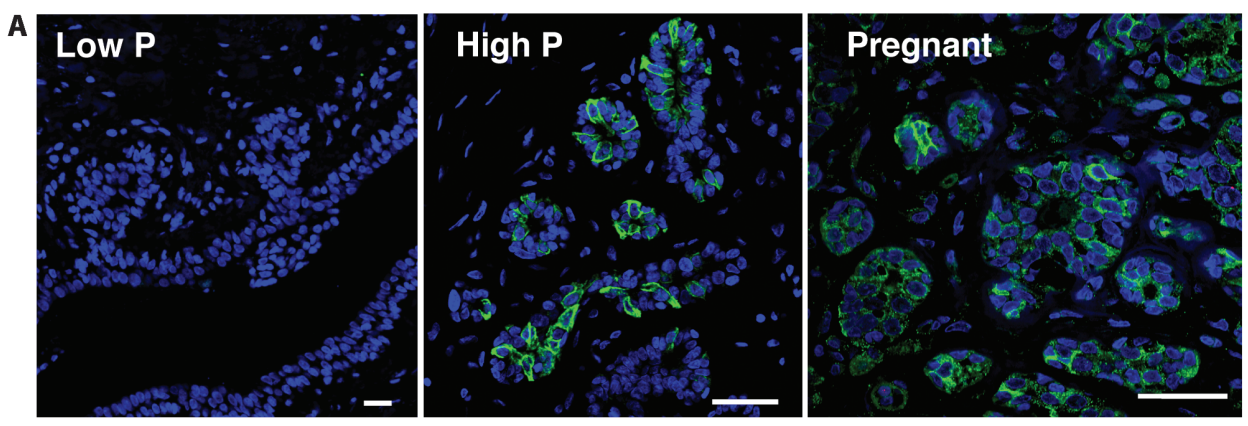

B
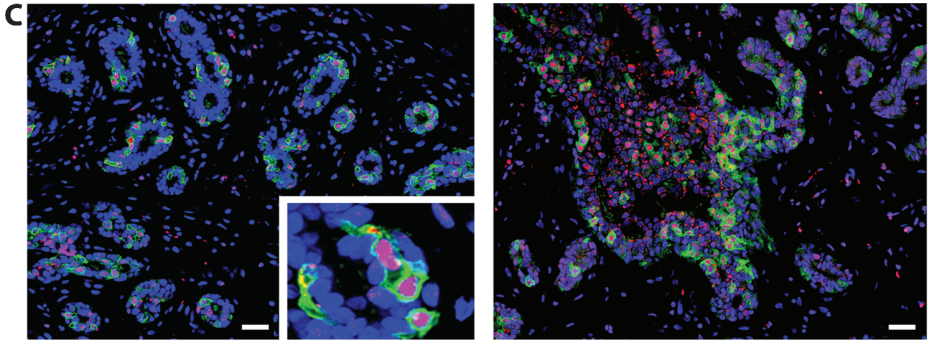

Fig. 5. In vivo RANKL expression and serum progesterone levels. (A) Human breast tissue sections stained with anti-RANKL antibody. Representative images of breast tissue sections from patients with low or high serum progesterone levels $(\mathrm{P})$ as well as from a pregnant patient. RANKL (green) and DAPI counterstain (blue). Scale bars, $100 \mu \mathrm{m}$. (B) Table showing number of human breast samples expressing detectable levels of RANKL

sufficient for cell proliferation. This indicates that a central hormonal control pathway is conserved between mouse and human, and suggests that at least some of the discrepancies reported between rodent and human models relate to differences between in vivo and in vitro approaches used for mouse and human, respectively. These findings have important clinical implications. Selective PR modulators are available, and a RANKL inhibitor, denosumab, has already been FDA-approved for treatment of postmenopausal women at high risk for fracture and for prevention of skeletal-related events in patients with bone metastases. The drugs may benefit breast cancer patients as preventive and therapeutic agents.

With the advent of powerful chromatin immunoprecipitation sequencing technology and the ENCODE project, most work on steroid hormone action has focused on mapping steroid receptor binding sites and transcriptional control mechanisms at the genome level (34). Our finding that a central physiological control pathway works, to substantial extent, by controlling mRNA stability suggests that posttranscriptional control mechanisms require more attention.

In examining potential clinical applications, it will be important to determine whether a wide spectrum of breast cancer patients may benefit from denosumab, or whether its use will be limited to any particular subtype of breast cancer (35). Considering the importance of repeated progesterone stimulation as it occurs with menstrual cycles as risk factor for breast cancer, the drug could, in particular, benefit premenopausal patients with high risk of getting breast cancer because it could abrogate the proliferative response elicited by the hormone. Especially young breast cancer patients, such as those less than 40 years old, who are at a 5\% risk of developing contralateral breast

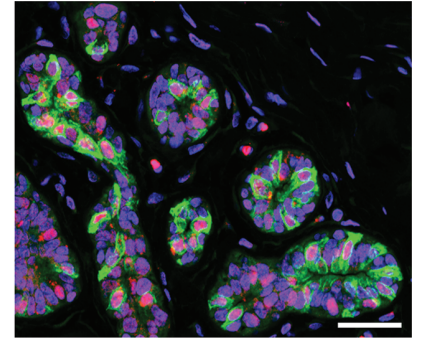

\begin{tabular}{|c|c|c|c|}
\hline & \multicolumn{2}{|c|}{ Nonpregnant } & Pregnant \\
\hline Progesterone levels & Low & High & High \\
\hline $\begin{array}{c}\text { RANKL } \\
\text { total samples/ }\end{array}$ & $0 / 10$ & $10 / 10$ & $2 / 2$ \\
\hline
\end{tabular}

D

\begin{tabular}{|c|c|c|}
\hline Age (years) & $\%$ RANKL ${ }^{+}$of $\mathrm{PR}^{+}$ & $\% \mathrm{PR}^{+}$of $\mathrm{RANKL}^{+}$ \\
\hline 51 & 78 & 98 \\
\hline 21 & 76 & 94 \\
\hline 44 & 78 & 99 \\
\hline 38 & 51 & 97 \\
\hline 27 & 55 & 98 \\
\hline 38 & 52 & 96 \\
\hline
\end{tabular}

protein over total number of samples analyzed in each group. (C) Coimmunofluorescence for PR (red) and RANKL (green) in samples from three patients with high serum progesterone levels. Scale bar, $50 \mu \mathrm{m}$. (D) Table showing the age of six different patients with high progesterone serum levels and the percentages of PR and RANKL coexpressing cells in their tissue samples.

cancer within 10 years (36), could benefit from blocking RANKL action during luteal phase and thus represent an excellent target group for a first clinical study.

The observation that $\mathrm{E}_{2}$ inhibits cell proliferation in the largest subgroup (group 1) is intriguing in light of recent studies that suggest a protective effect of estrogens against breast cancer risk (37). Yet, caution is required in interpreting the different responses to $\mathrm{E}_{2}$ and progesterone. Much larger cohorts will be required to discern how oral contraception, previous pregnancies, menopause, and other factors may impinge on the response.

Surprisingly, $E_{2}$, which induced PR expression, did not synergize with progesterone with regard to cell proliferation and RANKL induction. However, there is PR expression in the absence of exogenous $E_{2}$, and this may be sufficient for a maximal response to progesterone. Also, our expectations were based on experiments performed in ovariectomized rodents where the two hormones act synergistically to induce cell proliferation (38). Hormonally ablated mice and rats differ from endocrine-intact animals, which might more closely reflect the patients analyzed in the present study. Hormone signaling is extremely context-dependent, and there is increasing evidence for interactions between ER and PR at the molecular level that may account for this phenomenon (39).

The model reflects the context dependence that affects hormone signaling. Minimal treatment of the clinical specimens, simple culture conditions with use of basal medium, and no serum ensure that this assay is widely usable and reproducible. As such, the present model opens new avenues for unraveling mechanisms underlying the action of hormones and of hormonally active substances in the breast. 

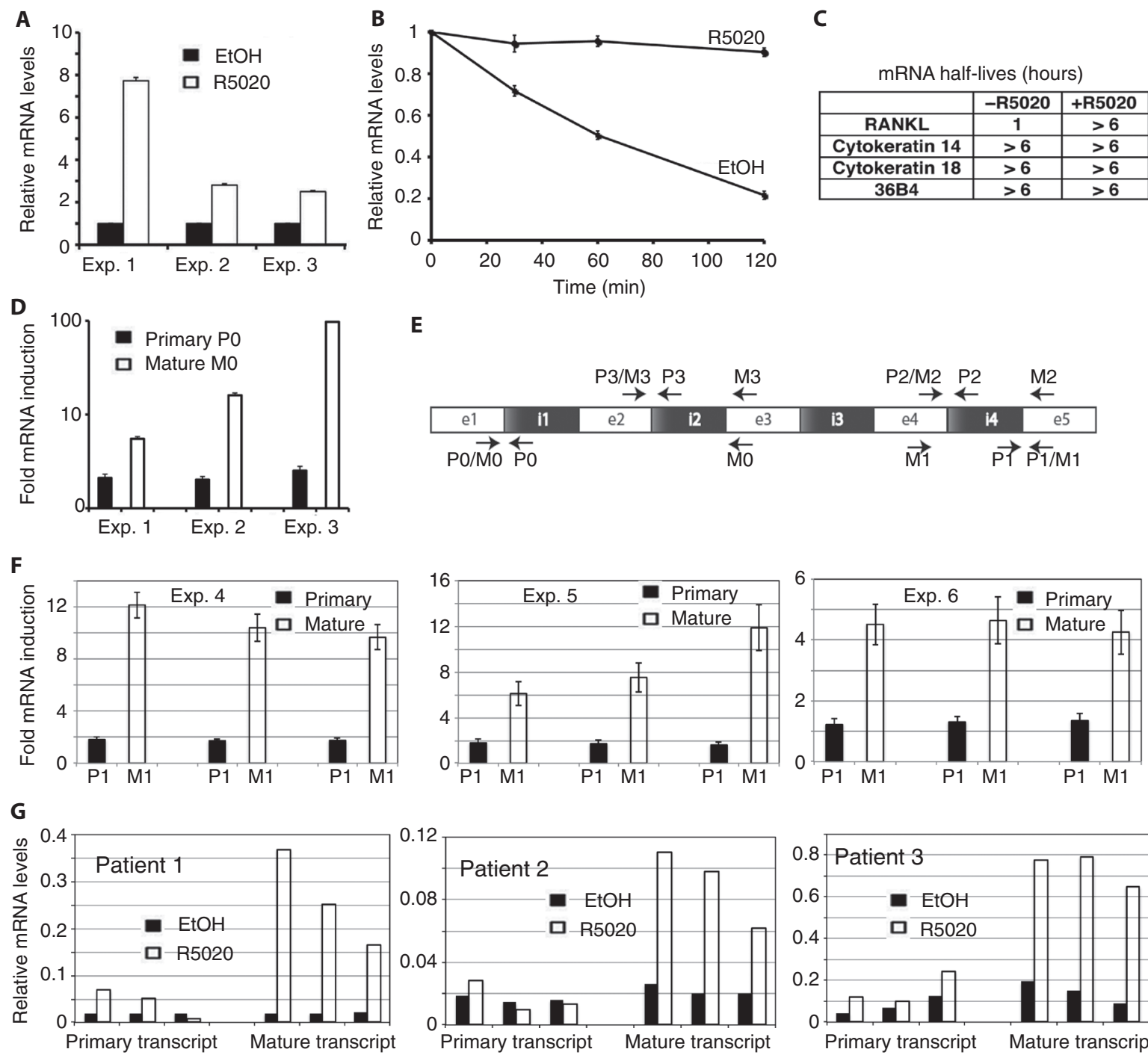

C

mRNA half-lives (hours)

\begin{tabular}{|c|c|c|}
\hline & - R5020 & +R5020 \\
\hline RANKL & 1 & $>6$ \\
\hline Cytokeratin 14 & $>6$ & $>6$ \\
\hline Cytokeratin 18 & $>6$ & $>6$ \\
\hline 36B4 & $>6$ & $>6$ \\
\hline \multicolumn{2}{|c}{} \\
\hline \multicolumn{2}{|c}{}
\end{tabular}

$\mathbf{E}$
Fig. 6. Control of RANKL mRNA expression by PR signaling. (A) Relative RANKL mRNA levels determined by qRT-PCR in freshly isolated mouse mammary organoids exposed for 30 min to R5020 or EtOH. Three independent experiments are shown; data represent means \pm SD of triplicates after normalization to TATA box-binding protein (TBP). (B) qRT-PCR of RANKL mRNA in primary mouse mammary epithelial cells at different times of actinomycin treatment in the presence or absence of R5020 plotted relative to initial value; data represent means $\pm S D$ of triplicates after normalization to TBP. (C) mRNA half-lives of RANKL and control mRNAs. (D) Ratio of RANKL primary and mature transcripts in mouse mammary organoids stimulated for 4 hours with R5020 versus EtOH only. Three

\section{MATERIALS AND METHODS}

\section{Patients, tissue samples, and processing}

The institutional ethics committee approved the study (45-05). To avoid bias, we obtained breast tissue from all women undergoing reduction mammoplasties from 2005 to 2012 with no previous history of breast cancer, who gave informed consent. All experiments were performed on fresh tissue. Replicates are on subsequent, hence random, mammoplasty specimens. independent experiments are shown; data represent means \pm SD of triplicates after normalization to TBP (primers P0 and M0). (E) Scheme of primer location within the mouse RANKL gene (e, exon; i, intron). (F) Fold induction of RANKL primary and mature transcripts with three distinct primer sets in three additional experiments in which mouse mammary organoids were stimulated for 4 hours with R5020 versus EtOH only. Data represent means \pm SD of triplicate measurements after normalization to TBP. (G) Levels of RANKL primary and mature transcripts determined by Fluidigm with three distinct primer sets in $\mathrm{EpCAM}^{+}$cells isolated by FACS from tissue microstructures from three different patients after 24 hours of treatment with $\mathrm{R} 5020$ or EtOH. Data were normalized to HPRT.

Samples were collected in the operating theater and confirmed by the pathologists to be free of malignancy. Tissue was minced manually to pieces of about $5-\mathrm{mm}$ diameter and digested overnight at $37^{\circ} \mathrm{C}$ with $2 \%$ collagenase A $(10 \mathrm{ml} / \mathrm{g})$ (Roche) in Dulbecco's modified Eagle's medium (DMEM)/F12. After enzymatic digestion, the tissue was centrifuged at $250 \mathrm{~g}$ for $4 \mathrm{~min}$ at $4^{\circ} \mathrm{C}$. Fat was removed, and the pellet containing tissue microstructures was washed with phosphatebuffered saline. 


\section{Tissue stimulation}

Freshly isolated tissue microstructures were stimulated with R5020 $(20 \mathrm{nM})$ and $\mathrm{E}_{2}(20 \mathrm{nM})$ (Sigma), RANKL $(1 \mu \mathrm{g} / \mathrm{ml})$, or OPG (10 or $20 \mu \mathrm{g} / \mathrm{ml}$ ) in phenol red-free, serum-free DMEM/F12 either during or after collagenase treatment.

\section{Development of a correlation network}

Network generation and integration of cell proliferation data were accomplished with Cytoscape software (http://www.cytoscape.org). The cell proliferation data were imported as fold change of the different pairwise comparisons of individual samples. One "node" in this network corresponds to an assayed sample, and the connection between them ("edge") refers to the correlation between the two nodes in the different experiments. Correlation networks were computed with the Cytoscape plug-in ExpressionCorrelation (http://www.baderlab. org/Software/ExpressionCorrelation), which facilitates assembly of a network; nodes with similar "behavior" (cell proliferation fold change) were connected and clustered on the basis of the Pearson correlation coefficient for all pairwise comparisons. A cutoff of 0.9 was used, so any correlations above this threshold value are displayed as an edge between two nodes. The edge thickness corresponds to the correlation coefficient level (0.9 to 0.99$)$. Nodes were colored with a red-to-blue gradient according to fold change proliferation index. Network images were generated with Cytoscape version 2.8.2 and exported to Adobe Illustrator for editing.

\section{Statistical analysis}

Statistical analysis was performed with $\mathrm{R}$ environment for box plots. For all other data, two-tailed, paired Student's $t$ test was used to calculate statistical significance; data are shown as means $\pm \mathrm{SD}$; $P$ values are indicated in the figures.

\section{SUPPLEMENTARY MATERIALS}

www.sciencetranslationalmedicine.org/cgi/content/full/5/182/182ra55/DC1 Materials and Methods

Fig. S1. Heterogeneity of human breast tissue.

Fig. S2. RANKL expression in different mammary cell populations.

Table S1. Reduction mammoplasty biobank.

\section{REFERENCES AND NOTES}

1. V. Beral; Million Women Study Collaborators, Breast cancer and hormone-replacement therapy in the Million Women Study. Lancet 362, 419-427 (2003).

2. C. Brisken, B. O'Malley, Hormone action in the mammary gland. Cold Spring Harb. Perspect. Biol. 2, a003178 (2010).

3. L. Ciarloni, S. Mallepell, C. Brisken, Amphiregulin is an essential mediator of estrogen receptor $\alpha$ function in mammary gland development. Proc. Natl. Acad. Sci. U.S.A. 104, 5455-5460 (2007).

4. S. Mallepell, A. Krust, P. Chambon, C. Brisken, Paracrine signaling through the epithelial estrogen receptor $\alpha$ is required for proliferation and morphogenesis in the mammary gland. Proc. Natl. Acad. Sci. U.S.A. 103, 2196-2201 (2006).

5. C. Brisken, S. Park, T. Vass, J. P. Lydon, B. W. O'Malley, R. A. Weinberg, A paracrine role for the epithelial progesterone receptor in mammary gland development. Proc. Natl. Acad. Sci. U.S.A. 95, 5076-5081 (1998).

6. D. L. Lacey, E. Timms, H. L. Tan, M. J. Kelley, C. R. Dunstan, T. Burgess, R. Elliott, A. Colombero, G. Elliott, S. Scully, H. Hsu, J. Sullivan, N. Hawkins, E. Davy, C. Capparelli, A. Eli, Y. X. Qian, S. Kaufman, I. Sarosi, V. Shalhoub, G. Senaldi, J. Guo, J. Delaney, W. J. Boyle, Osteoprotegerin ligand is a cytokine that regulates osteoclast differentiation and activation. Cell 93, 165-176 (1998).
7. J. E. Fata, Y. Y. Kong, J. Li, T. Sasaki, J. Irie-Sasaki, R. A. Moorehead, R. Elliott, S. Scully, E. B. Voura, D. L. Lacey, W. J. Boyle, R. Khokha, J. M. Penninger, The osteoclast differentiation factor osteoprotegerin-ligand is essential for mammary gland development. Cell 103, 41-50 (2000).

8. M. Beleut, R. D. Rajaram, M. Caikovski, A. Ayyanan, D. Germano, Y. Choi, P. Schneider, C. Brisken, Two distinct mechanisms underlie progesterone-induced proliferation in the mammary gland. Proc. Natl. Acad. Sci. U.S.A. 107, 2989-2994 (2010).

9. A. Mukherjee, S. M. Soyal, J. Li, Y. Ying, B. He, F. J. DeMayo, J. P. Lydon, Targeting RANKL to a specific subset of murine mammary epithelial cells induces ordered branching morphogenesis and alveologenesis in the absence of progesterone receptor expression. FASEB J. 24, 4408-4419 (2010).

10. M. L. Asselin-Labat, F. Vaillant, J. M. Sheridan, B. Pal, D. Wu, E. R. Simpson, H. Yasuda, G. K. Smyth T. J. Martin, G. J. Lindeman, J. E. Visvader, Control of mammary stem cell function by steroid hormone signalling. Nature 465, 798-802 (2010).

11. P. A. Joshi, H. W. Jackson, A. G. Beristain, M. A. Di Grappa, P. A. Mote, C. L. Clarke, J. Stingl, P. D. Waterhouse, R. Khokha, Progesterone induces adult mammary stem cell expansion. Nature 465, 803-807 (2010).

12. M. Shackleton, F. Vaillant, K. J. Simpson, J. Stingl, G. K. Smyth, M. L. Asselin-Labat, L. Wu, G. J. Lindeman, J. E. Visvader, Generation of a functional mammary gland from a single stem cell. Nature 439, 84-88 (2006).

13. J. Stingl, P. Eirew, I. Ricketson, M. Shackleton, F. Vaillant, D. Choi, H. I. Li, C. J. Eaves, Purification and unique properties of mammary epithelial stem cells. Nature 439, 993-997 (2006).

14. A. Van Keymeulen, A. S. Rocha, M. Ousset, B. Beck, G. Bouvencourt, J. Rock, N. Sharma, S. Dekoninck, C. Blanpain, Distinct stem cells contribute to mammary gland development and maintenance. Nature 479, 189-193 (2011).

15. R. van Amerongen, A. N. Bowman, R. Nusse, Developmental stage and time dictate the fate of Wnt/ $\beta$-catenin-responsive stem cells in the mammary gland. Cell Stem Cell 11, 387-400 (2012).

16. E. Gonzalez-Suarez, A. P. Jacob, J. Jones, R. Miller, M. P. Roudier-Meyer, R. Erwert, J. Pinkas, D. Branstetter, W. C. Dougall, RANK ligand mediates progestin-induced mammary epithelial proliferation and carcinogenesis. Nature 468, 103-107 (2010).

17. D. Schramek, A. Leibbrandt, V. Sigl, L. Kenner, J. A. Pospisilik, H. J. Lee, R. Hanada, P. A. Joshi, A. Aliprantis, L. Glimcher, M. Pasparakis, R. Khokha, C. J. Ormandy, M. Widschwendter, G. Schett, J. M. Penninger, Osteoclast differentiation factor RANKL controls development of progestindriven mammary cancer. Nature 468, 98-102 (2010).

18. W. Tan, W. Zhang, A. Strasner, S. Grivennikov, J. Q. Cheng, R. M. Hoffman, M. Karin, Tumourinfiltrating regulatory $T$ cells stimulate mammary cancer metastasis through RANKL-RANK signalling. Nature 470, 548-553 (2011).

19. T. Tanos, L. J. Rojo, P. Echeverria, C. Brisken, ER and PR signaling nodes during mammary gland development. Breast Cancer Res. 14, 210 (2012).

20. R. Fernandez-Valdivia, A. Mukherjee, C. J. Creighton, A. C. Buser, F. J. DeMayo, D. P. Edwards, J. P. Lydon, Transcriptional response of the murine mammary gland to acute progesterone exposure. Endocrinology 149, 6236-6250 (2008).

21. C. D. Roskelley, P. Y. Desprez, M. J. Bissell, Extracellular matrix-dependent tissue-specific gene expression in mammary epithelial cells requires both physical and biochemical signal transduction. Proc. Natl. Acad. Sci. U.S.A. 91, 12378-12382 (1994).

22. L. Kass, J. T. Erler, M. Dembo, V. M. Weaver, Mammary epithelial cell: Influence of extracellular matrix composition and organization during development and tumorigenesis. Int. J. Biochem. Cell Biol. 39, 1987-1994 (2007).

23. J. D. Graham, P. A. Mote, U. Salagame, J. H. van Dijk, R. L. Balleine, L. I. Huschtscha, R. R. Reddel, C. L. Clarke, DNA replication licensing and progenitor numbers are increased by progesterone in normal human breast. Endocrinology 150, 3318-3326 (2009).

24. R. D. Rajaram, C. Brisken, Paracrine signaling by progesterone. Mol. Cell. Endocrinol. 357 80-90 (2012).

25. A. Ayyanan, O. Laribi, S. Schuepbach-Mallepell, C. Schrick, M. Gutierrez, T. Tanos, G. Lefebvre, J. Rougemont, O. Yalcin-Ozuysal, C. Brisken, Perinatal exposure to bisphenol a increases adult mammary gland progesterone response and cell number. Mol. Endocrinol. 25, 1915-1923 (2011).

26. E. M. McGowan, N. Alling, E. A. Jackson, D. Yagoub, N. K. Haass, J. D. Allen, R. Martinello-Wilks, Evaluation of cell cycle arrest in estrogen responsive MCF-7 breast cancer cells: Pitfalls of the MTS assay. PLoS One 6, e20623 (2011).

27. R. B. Clarke, A. Howell, C. S. Potten, E. Anderson, Dissociation between steroid receptor expression and cell proliferation in the human breast. Cancer Res. 57, 4987-4991 (1997).

28. M. E. Smoot, K. Ono, J. Ruscheinski, P. L. Wang, T. Ideker, Cytoscape 2.8: New features for data integration and network visualization. Bioinformatics 27, 431-432 (2011).

29. P. C. Echeverría, F. Forafonov, D. P. Pandey, G. Mühlebach, D. Picard, Detection of changes in gene regulatory patterns, elicited by perturbations of the $\mathrm{Hsp} 90$ molecular chaperone complex, by visualizing multiple experiments with an animation. BioData Min. 4, 15 (2011).

30. E. Bachelard-Cascales, M. Chapellier, E. Delay, G. Pochon, T. Voeltzel, A. Puisieux, C. Caron de Fromentel, V. Maguer-Satta, The CD10 enzyme is a key player to identify and regulate human mammary stem cells. Stem Cells 28, 1081-1088 (2010). 
31. C. Brisken, A. Heineman, T. Chavarria, B. Elenbaas, J. Tan, S. K. Dey, J. A. McMahon, A. P. McMahon R. A. Weinberg, Essential function of Wnt-4 in mammary gland development downstream of progesterone signaling. Genes Dev. 14, 650-654 (2000).

32. J. R. Masters, J. O. Drife, J. J. Scarisbrick, Cyclic variation of DNA synthesis in human breast epithelium. J. Natl. Cancer Inst. 58, 1263-1265 (1977).

33. B. Mulac-Jericevic, J. P. Lydon, F. J. DeMayo, O. M. Conneely, Defective mammary gland morphogenesis in mice lacking the progesterone receptor B isoform. Proc. Natl. Acad. Sci. U.S.A. 100, 9744-9749 (2003).

34. C. S. Ross-Innes, R. Stark, A. E. Teschendorff, K. A. Holmes, H. R. Ali, M. J. Dunning, G. D. Brown, O. Gojis, I. O. Ellis, A. R. Green, S. Ali, S. F. Chin, C. Palmieri, C. Caldas, J. S. Carroll, Differential oestrogen receptor binding is associated with clinical outcome in breast cancer. Nature $\mathbf{4 8 1}$ 389-393 (2012).

35. C. Curtis, S. P. Shah, S. F. Chin, G. Turashvili, O. M. Rueda, M. J. Dunning, D. Speed, A. G. Lynch S. Samarajiwa, Y. Yuan, S. Gräf, G. Ha, G. Haffari, A. Bashashati, R. Russell, S. McKinney; METABRIC Group, A. Langerød, A. Green, E. Provenzano, G. Wishart, S. Pinder, P. Watson, F. Markowetz L. Murphy, I. Ellis, A. Purushotham, A. L. Børresen-Dale, J. D. Brenton, S. Tavaré, C. Caldas, S. Aparicio, The genomic and transcriptomic architecture of 2,000 breast tumours reveals novel subgroups. Nature 486, 346-352 (2012).

36. M. Hartman, K. Czene, M. Reilly, J. Bergh, P. Lagiou, D. Trichopoulos, H. O. Adami, P. Hall, Genetic implications of bilateral breast cancer: A population based cohort study. Lancet Oncol. 6, 377-382 (2005).

37. G. L. Anderson, R. T. Chlebowski, A. K. Aragaki, L. H. Kuller, J. E. Manson, M. Gass, E. Bluhm, S. Connelly, F. A. Hubbell, D. Lane, L. Martin, J. Ockene, T. Rohan, R. Schenken, J. WactawskiWende, Conjugated equine oestrogen and breast cancer incidence and mortality in postmenopausal women with hysterectomy: Extended follow-up of the Women's Health Initiative randomised placebo-controlled trial. Lancet Oncol. 13, 476-486 (2012).

38. T. K. Said, O. M. Conneely, D. Medina, B. W. O'Malley, J. P. Lydon, Progesterone, in addition to estrogen, induces cyclin D1 expression in the murine mammary epithelial cell, in vivo. Endocrinology 138, 3933-3939 (1997).

39. S. Giulianelli, J. P. Vaqué, R. Soldati, V. Wargon, S. I. Vanzulli, R. Martins, E. Zeitlin, A. A. Molinolo, L. A. Helguero, C. A. Lamb, J. S. Gutkind, C. Lanari, Estrogen receptor alpha mediates progestininduced mammary tumor growth by interacting with progesterone receptors at the cyclin D1/MYC promoters. Cancer Res. 72, 2416-2427 (2012).
40. L. K. Swee, K. Ingold-Salamin, A. Tardivel, L. Willen, O. Gaide, M. Favre, S. Demotz, M. Mikkola, P. Schneider, Biological activity of ectodysplasin A is conditioned by its collagen and heparan sulfate proteoglycan-binding domains. J. Biol. Chem. 284, 27567-27576 (2009).

41. P. Schneider, Production of recombinant TRAIL and TRAIL receptor: Fc chimeric proteins. Methods Enzymol. 322, 325-345 (2000).

Acknowledgments: We thank M. Bueno, M. Garcia, G. Tapia (Ecole Polytechnique Fédérale de Lausanne), and L. Willen (University of Lausanne) for technical assistance. Funding: Supported by National Center of Competence in Research Molecular Oncology, Oncosuisse, SNF-3100AO112090 (C.B.), SNF-31003-138065 (P.S.), and Marie-Curie incoming fellowship (T.T.). The research leading to these results has received support from the Innovative Medicines Initiative Joint Undertaking under grant agreement no. 115188, resources of which are composed of financial contribution from the European Union's Seventh Framework Programme (FP7/20072013) and European Federation of Pharmaceutical Industries and Associations companies' in kind contribution. The Web address of Innovative Medicines Initiative is http://www.imi.europa.eu/ Author contributions: T.T., G.S., A.A., and M.G. performed the experiments; T.T. and G.S. analyzed the experiments; P.C.E. performed Cytoscape analysis; W.D. and P.S. provided reagents and input on the manuscript; O.Y.-O. designed the experiments and analyzed the data; W.R., J.-F.D. and M.F. helped with clinical samples; and C.B. designed study and wrote the manuscript. Competing interests: W.D. is an employee of Amgen Inc. and has a financial relationship with Amgen Inc. including equity holdings and stock options. The other authors declare that they have no competing interests.

Submitted 7 January 2013

Accepted 27 February 2013

Published 24 April 2013

$10.1126 /$ scitranslmed.3005654

Citation: T. Tanos, G. Sflomos, P. C. Echeverria, A. Ayyanan, M. Gutierrez, J.-F. Delaloye, W. Raffoul, M. Fiche, W. Dougall, P. Schneider, O. Yalcin-Ozuysal, C. Brisken, Progesterone/ RANKL is a major regulatory axis in the human breast. Sci. Transl. Med. 5, 182ra55 (2013). 